\title{
Origin of GRB afterglows in the model of galactic neutron stars
}

\author{
G.S. Bisnovatyi-Kogan \\ Space Research Institute, 84/32, Profsoyuznaya st., Moscow, Russia \\ e-mail: gkogan@mx.iki.rssi.ru
}

Received December 29, 1998; accepted June 3, 1999

\begin{abstract}
The launch of the Beppo-Sax satellite gave a unique opportunity to investigate gamma ray bursts (GRB) in different spectral regions. The large diversity of the afterglow behavior creates additional problems for the cosmological model with a fireball. Formation of the afterglow giving the observed diversity of properties is suggested in the Galactic neutron star model of GRBs. It is based on the transient accretion disc formation around the neutron star with a low-mass brown companion irradiated by the neutron star.
\end{abstract}

Key words: gamma-ray: bursts

\section{Introduction}

A cosmological origin of GRBs leads to the conclusion of a huge energy output. If the identification with the galaxy having redshift $z=3.42$ is true, then in gamma radiation the energy release (without beaming) is $\sim 310^{53} \mathrm{ergs}$ (Kulkarni 1998). Note, that the solar rest mass is equal to $1.810^{54}$ ergs. Small timescale indicates that GRBs are related to neutron stars and (or) stellar mass black holes. The common event from a collapse with a neutron star formation is a supernova explosion. The total energy release $E_{\text {tot }}$ in a $\mathrm{SN}$ is equal to the binding energy of a neutron star $\sim 20 \% \mathrm{Mc}^{2}$. For the neutron star with a mass $1.4 M_{\odot}$ we get $E_{\text {tot }} \approx 510^{53}$ ergs, which is comparable to the above estimate for a GRB. Only a small part $\sim 310^{51} \mathrm{ergs}$ is transformed into kinetic energy of the explosion, and the energy radiated in all parts of the electromagnetic spectrum is several tens times less. More then $99 \%$ of the total energy output is emitted in the form of weakly interacting neutrinos, and is dispersed in the Universe. An artificially constructed low-temperature structure around a neutron star or a black hole may suffer from an instability. Stars with a neutron core (Thorne-Zytkov model) are in most cases unstable to run-away neutrino emission, leading to radiation by neutrinos of more then $99 \%$ of

Send offprint requests to: G.S. Bisnovatyi-Kogan the accretion energy. Magnetorotational explosion, used by Pazcynski (1998) to explain the huge energy production in a cosmological GRB, had been suggested for the supernova explosion by Bisnovatyi-Kogan (1971). Numerical 1-D and 2-D calculations gave the efficiency of a transformation of the rotational energy into the kinetic one at the level of few percent (Ardelyan et al. 1997). The restrictions of the "hypernova" model of Pazcynski (1998) had been analyzed by Blinnikov \& Postnov (1998). The total explosive energy output at the end of the evolution of a close binary, consisting of two neutron stars, suggested for a GRB model by Blinnikov at al. (1984) cannot exceed the value of a (positive) binding energy less than $10^{-3} M_{\odot} c^{2}=1.610^{51}$ ergs (Saakyan \& Vartanyan 1964). Only part of this energy may be radiated in the GRB region. In the presence of serious energy problems inherent in the model of the cosmological GRB, we try to explain the pioneering results of the afterglow measurements by Beppo-Sax, as well as previous hard gamma-ray afterglow observed by EGRET, in the frame of the model of GRB origin in the old nearby neutron stars inside the Galactic disk. The host galaxies with a high redshift are supposed to be a chance coincidence with GRB. Isotropy on the sky and non $3 / 2 \log N / \log S$ may be connected with selection effects (Bisnovatyi-Kogan 1997), or a local non-uniformity (B.V. Komberg, priv. comm.).

\section{The model and neutron star statistics}

We consider a gamma-ray burst model based on a nuclear explosion under the surface of a neutron star. There is a nonequilibrium layer in the crust of the neutron star, consisting of very heavy and neutron overabundant nuclei. After the starquake these nuclei are moved out, and become unstable to fission after several beta-decays. This results in an almost instant explosion and the formation of a GRB (Bisnovatyi-Kogan et al. 1975). The energy resource in the nonequilibrium layer is of the order of $10^{47}$ ergs. If GRB originates at a distance about 100 pc with the energy release $10^{36}-10^{40}$ ergs, then each neutron star may give $10^{7}-10^{11}$ gamma ray bursts. Estimating the total 
number of neutron stars in the Galaxy as $(2-7) 10^{8}$, and their number inside the sphere with a radius $200 \mathrm{pc}$ as $(3-10) 10^{4}$, we need a recurrence time $(100-300)$ years in old neutron stars to produce an observed GRB every day (Bisnovatyi-Kogan 1992).

\section{Hard gamma-ray afterglow}

The 1.5 hours afterglow observed by EGRET in GRB of 17 February 1994 in the energy band $(40-18000) \mathrm{MeV}$ has a simple explanation in the local model. A nuclear explosion in the neutron star crust excites eigen-oscillations in the neutron star with periods $(0.1-10) \mathrm{ms}$. In presence of such high frequency oscillations the old slowly rotating neutron star with a relatively small magnetic field becomes a transient high frequency radio pulsar. The radio pulsars are known as strongest sources in the hard gamma ray band of EGRET, so the observed hard gamma ray afterglow could be related to the activity of this transient high frequency radio pulsar (Bisnovatyi-Kogan 1995, 1997). To test this scheme it was suggested to perform high time resolution radio observations of the Vela pulsar shortly after the observed glitch, which happen almost every year. This proposal (Bisnovatyi-Kogan \& Tsarevsky 1998) is now under consideration.

\section{X-ray, optical and radio afterglows}

Beppo-Sax observations gave the X-ray flux for GRB afterglow on the level $\sim 10^{-13} \mathrm{ergs} / \mathrm{s}$. Taking 3 days for the duration of X-ray emission we get $F_{\mathrm{x}} \sim 310^{-8} \mathrm{ergs} / \mathrm{cm}^{2}$ for the $\mathrm{X}$-ray fluence, which gives $\sim 1 / 30$ of the main GRB with total fluency $\sim 10^{-6} \mathrm{ergs} / \mathrm{cm}^{2}$. Explosion on the neutron star could lead to nonradial mass ejection with a velocity between escape and Keplerian velocities. The ejected matter falls back to the neutron star forming an accretion disk due to its high angular momentum. Taking the distance $\sim 100 \mathrm{pc}$, corresponding to the total GRB energy $E_{\gamma} \sim 10^{36} \mathrm{ergs}$, and $E_{\mathrm{x}} \sim 310^{34} \mathrm{ergs}$, we estimate the mass creating this $\mathrm{X}$-ray flux during accretion into a neutron star as $10^{-19} M_{\odot}$. The spectrum of accretion with a low rate $\dot{M} \sim 310^{-17} M_{\odot} /$ yr $\approx 710^{8} \mathrm{~g} / \mathrm{s}$, consist of two approximately equal parts. The first is a relatively hard $\mathrm{X}$-ray emission in the range $1-10 \mathrm{keV}$ from the boundary layer. The second is the radiation from the accretion disk itself which emits a spectrum $F_{\gamma} \sim \omega^{1 / 3}$ with an exponential cutoff starting from $\omega \approx 210^{15} \mathrm{~s}^{-1}$, corresponding to a maximum temperature $210^{4} \mathrm{~K}$, which color corresponds to a $B$ star. Duration of the emission from the accretion disk is not expected to last a long time, and it is not expected to radiate much in the red and far red ranges, lasting several months (Sokolov 1998).

The extended GRB afterglows with spectra corresponding to a cold star may be explained easily if the neutron star has a low mass companion with $M_{\mathrm{d}}=$ $(0.02-0.2) M_{\odot}$, as observed in most binary recycled pulsars. Taking a companion with a mass $0.03 M_{\odot}$ and very low temperature (degenerate brown dwarf) with a normal composition $\left(R_{\mathrm{d}} \sim 5.310^{9} \mathrm{~cm}\right)$, or without hydrogen $\left(R_{\mathrm{d}} \sim 2.210^{9} \mathrm{~cm}\right)$, and binary separation $R_{12} \sim 10^{11} \mathrm{~cm}$, we get a time for binary merging due to a gravitational radiation $\tau_{\mathrm{g}}=\frac{5 c^{5} R_{12}^{4}}{256 G^{3} M_{\mathrm{d}} M_{\mathrm{n}}\left(M_{\mathrm{d}}+M_{\mathrm{n}}\right)}=$ $210^{16} \frac{R_{11}^{4}}{\left(M_{\mathrm{d}} M_{\mathrm{n}}\left(M_{\mathrm{d}}+M_{\mathrm{n}}\right)\right)_{\odot}}$ s of the order of a cosmological time. This companion absorbs $\sim\left(R_{\mathrm{d}} / R_{12}\right)^{2}$ of the total energy flux, what is equal to $\sim 0.003, \sim 0.0005$ for normal composition, and hydrogen-free dwarfs relatively. To obtain an afterglow with energy comparable with the energy of the observed GRB we should imagine that efficiency of GR production in the event does not exceed $\sim 1 \%$, and the main energy is radiated in the form of the kinetic energy, or relativistic particles. It corresponds to the total energy output $\sim 10^{38}$ ergs. The shock wave may heat the surface to high temperatures, leading to an X-ray flash $\sim\left(R_{12} / c\right) \approx 3$ seconds after the main GRB. For a longer GRB it means the corresponding change of the GRB spectrum with a sharp rise in the soft part. Radiation flux and ultrarelativistic particles penetrate deeper under the surface of the star heating a rather thick layer. Taking the absorption cross-section $\sim 10^{-26} \mathrm{~cm}^{2}$, and the absorbed flux $(1-3) 10^{35} \mathrm{ergs}$, corresponding to $\sim 10^{16} \mathrm{ergs} / \mathrm{cm}^{2}$, we obtain a surface temperature $\sim 10^{5} \mathrm{~K}$ immediately after absorption. It means that relatively strong ultraviolet source appears, accompanied by a strong mass loss feeding additionally the accretion disk around the neutron star. After a short $(\sim 10$ seconds $)$ phase of the mass loss and UV emission the temperature drops, and a week cooling object appears with a spectrum moving into the red and IR region, which is in general accordance with the observed afterglows.

\section{References}

Ardelyan N.V., Bisnovatyi-Kogan G.S., Moiseenko S.G., 1997, Physics-Uspekhi 40, 1076

Bisnovatyi-Kogan G.S., 1971, Sov. Astron. 14, 652

Bisnovatyi-Kogan G.S., 1995, ApJS 97, 185

Bisnovatyi-Kogan G.S., 1997, A\&A 324, 573

Bisnovatyi-Kogan G.S., 1992, Proc. IAU Symp. "The Stellar Population of Galaxies", Barbuy B. and Renzini A. (eds.). Kluwer, p. 379

Bisnovatyi-Kogan G.S., Imshennik V.S., Nadyozhin D.K., Chechetkin V.M., 1975, Ap\&SS 35, 23

Bisnovatyi-Kogan G.S., Tsarevsky G.S., 1998, Proc. JENAM 98 (in press)

Blinnikov S.I., Novikov I.D., Perevodchikova T.V., Polnarev A.G., 1984, Sov. Astron. Lett. 10, 177

Blinnikov S.I., Postnov K.A., 1998, MNRAS 293, L29

Kulkarni S., 1998 (this volume)

Paczynski B., 1998, ApJ 494, L45

Saakyan G.S., Vartanyan Yu.L., 1964, Astron. Zh. 41, 193

Sokolov V.V., 1998 (this volume) 